\title{
La Société nationale française de proctologie (SNFCP) : une société ouverte, un outil de travail et de formation médicale continue
}

\author{
A.-L. Tarrerias \\ (C) Springer-Verlag France 2010
}

La proctologie chirurgicale pratiquée par les gastroentérologues fait face, depuis quelques mois, à une augmentation brutale des primes d'assurance avec des montants parfois inadaptés aux tarifs appliqués à nos actes. La SNFCP a donc, grâce à l'appui du Synmad, commencé un travail avec la MACSF dans le but de comprendre cette dérive et de l'éviter. Il s'avère qu'au vu des dossiers en cours, le nombre de procédures n'a pas augmenté, que les patients sont le plus souvent déboutés, leurs symptômes n'étant pas secondaires à leur intervention ou encore parce qu'aucune faute n'a été reconnue. En revanche, quand les patients sont déboutés, le praticien est condamné à chaque fois que l'information et le consentement éclairé n'étaient pas recueillis, le tribunal considérant comme une perte de chance pour le patient de n'avoir pu envisager de renoncer à l'intervention. L'inquiétude des assureurs vient de l'augmentation des montants réclamés pour l'indemnisation des patients, plus que du nombre de procédures.

Le bureau de la SNFCP a donc fait un important travail de rédaction de fiches d'informations concernant les interventions de proctologie les plus courantes. Ces fiches, estampillées du logo de la SNFCP, vont paraître prochainement sur le site. Vous pourrez, pour chaque intervention, remettre la fiche d'information correspondant à l'intervention que vous programmez et, sur un autre document, un consentement éclairé type que vous trouverez aussi sur le site.
L'information doit être remise au patient, le consentement éclairé du patient doit être récupéré avant l'intervention. Il n'est plus acceptable, compte tenu de la jurisprudence actuelle, de se passer de cette procédure, d'autant que ces documents sont maintenant faciles d'accès. Les fiches d'informations ont été rédigées par les membres du bureau de la SNFCP, elles ont été validées par la MACSF.

Ce travail permet de ramener la prime d'assurance de la proctologie chirurgicale à un niveau équivalent à celui de l'endoscopie interventionnelle.

Ce long travail en commun entre le bureau de la SNFCP et la MACSF sera détaillé lors de l'ouverture des journées du 26 et 27 novembre 2010, et le Dr D. Neuveu, gastroentérologue de la MACSF, nous éclairera en matière de procédures en cours dans le domaine de la chirurgie de l'anus.

Le site de la SNFCP se modernise pour devenir un outil professionnel mais aussi de formation. Les conférences de notre dernier congrès, en novembre 2009, sont disponibles sous forme de podcasts téléchargeables. Si vous avez manqué ce congrès, n'hésitez pas à consulter tous les sujets et/ou à les télécharger sur votre iPod. Notre prochain congrès aura lieu les 26 et 27 novembre 2010 aux salons d'Iéna. Vous pouvez vous inscrire dès maintenant sur le site de la SNFCP : www.snfcp.org. Comme l'année dernière, vous pourrez pratiquer les scléroses, ligatures, HAL (ligature d'hémorroïdes sous doppler), et longo sur les endotrainers (inscription le vendredi matin à l'ouverture du congrès).

A.-L. Tarrerias $(\bowtie)$

SNFCP, Institut Mutualiste Montsouris,

42, boulevard Jourdan,

F-75674 Paris cedex 14, France

e-mail : al.tarrerias@wanadoo.fr 